\title{
Origins of the Exchange-Bias Phenomenology, Coercivity Enhancement, and Asymmetric Hysteretic Shearing in Core-Surface Smart Nanoparticles
}

\author{
Rıza Erdem, ${ }^{1}$ Orhan Yalçın, ${ }^{2}$ Songüil Özüim, ${ }^{3}$ and Nazire Çiftçi ${ }^{4}$ \\ ${ }^{1}$ Department of Physics, Akdeniz University, 07058 Antalya, Turkey \\ ${ }^{2}$ Department of Physics, Niğde University, 51240 Niğde, Turkey \\ ${ }^{3}$ Institute of Science, Niğde University, 51240 Niğde, Turkey \\ ${ }^{4}$ Institute of Science, Gaziosmanpaşa University, 60240 Tokat, Turkey
}

Correspondence should be addressed to Riza Erdem; rerdem@akdeniz.edu.tr

Received 15 March 2016; Revised 28 June 2016; Accepted 18 July 2016

Academic Editor: Da-Ren Hang

Copyright (C) 2016 Riza Erdem et al. This is an open access article distributed under the Creative Commons Attribution License, which permits unrestricted use, distribution, and reproduction in any medium, provided the original work is properly cited.

\begin{abstract}
We have used a spin-1 Ising model Hamiltonian with dipolar (bilinear, $J$ ), quadrupolar (biquadratic, $K$ ), and dipolar-quadrupolar (odd, $L$ ) interactions in pair approximation to investigate the exchange-bias (EB), coercive field, and asymmetric hysteretic shearing properties peculiar to core/surface $(C / S)$ composite nanoparticles (NPs). Shifted hysteresis loops with an asymmetry and coercivity enhancement are observed only in the presence of the odd interaction term in the Hamiltonian expression and their magnitudes show strong dependence on the value of $L$. The observed coercivity and EB in $C / S$ NPs originated from nonzero odd coupling energies and their dependence on temperature $(T)$ and particle size $(R)$ are also discussed in relation to experimental findings.
\end{abstract}

\section{Introduction}

The exchange-bias (EB) phenomenon is defined as shift of the hysteresis loop along magnetic field $(h)$ axis, in either negative or positive direction. It is one of the phenomena associated with the exchange anisotropy created at the interface between ferromagnetic (FM) and antiferromagnetic (AFM) spin structures. The anisotropy was first discovered by Meiklejohn and Bean [1]. Since then, it was observed in many different systems containing FM/AFM interfaces, such as layered thin films [2], inhomogeneous materials [3], and small particles [4]. Most of the subsequent studies [517] have been focused on exchange-biased nanostructures composed of FM/AFM interfaces because of their technological applications in advanced magnetic devices. In particular, the temperature and size dependence of $\mathrm{EB}$ in FM/AFM nanoparticles (NPs), including commonly FM (core)/AFM (shell) interface, has attracted a lot of attention [8-11, 15].

However, only very recently, some works partially addressing the numerical studies of $\mathrm{EB}$ effects in magnetic
NPs have been published [18-24]. In order to understand the origin of the loop shifts displayed numerically in NPs with core-shell morphology, the researchers simulated, using the Monte Carlo (MC) technique, the atomic-scale modeling where the spins interact with nearest-neighbor Heisenberg interactions. From the cooling field strength versus EB field $\left(h_{E}\right)$ and coercive field $\left(h_{C}\right)$ variations, their simulations have shown that the asymmetry in the hysteresis loops was due to the different magnetization reversal mechanisms in two branches related to exchange coupling at the FM/AFM interface $[18,19]$. On the other hand, a variety of experimental facts associated with EB for the coupled FM/AFM layers were modeled as Ising-type system due to their strong uniaxial anisotropy [25-28]. Within the mean-field-type calculations, a shift of the hysteresis loop along the magnetic field axis was accompanied by an enhancement of the coercivity [27].

While there is vast literature on this enhancement of $h_{C}$ in finite and infinite systems (for a review see [29]), less attention has been paid to Ising model formulation in pair approximation (PA) for the appearance of EB in NPs systems 
[30]. This theory treats the nearest-neighbor pair correlations between Ising spins within the nanoparticle explicitly, and therefore being capable of describing core/surface $(C / S)$ composite NPs. For these particles the correlations between nearest-neighbors are significant to establish boundaries between core $(C)$ and surface $(S)$ parts with different magnetic properties. A similar approach has recently been used by us to investigate the hysteretic splitting of $C / S$ NPs $[31,32]$. In the presence of an external magnetic field, we have taken into account only even interactions (or dipolar, quadrupolar, and single-ion anisotropy) between Ising spins in $C$, interface $(C S)$, and $S$ parts within the nanoparticle. The microscopic model that uses these properties is called the Blume-Emery-Griffiths (BEG) model [33]. It works well for systems characterized with fluctuations in both density and magnetic ordering when the odd sector contributions (or dipolar-quadrupolar interaction, external magnetic field) are included in the Hamiltonian. For a complete description of phase transitions arising in bulk systems, the full BEG Hamiltonian with both even and odd interaction terms was studied using various techniques [34-39]. But the odd term has not been considered for the analysis of structural transitions in NPs presented in [31, 32]. By incorporating odd interaction the PA formalism may provide a theoretical framework for the EB effect and the asymmetry of the hysteresis loops observed in experiments made on a single (or independent) composite nanoparticle.

In this work, we will use, in addition to the even interactions, odd sector contribution to the Ising Hamiltonian in order to obtain a complete description of magnetic and hysteretic properties for the smart C/S NPs. Particularly, we will show, through PA calculations, how the EB field observed in the smart $C / S$ NPs is related to this contribution. Moreover, the direct inspection of magnetization along the asymmetric hysteresis loops will allow us to provide quantitative understanding of these loops and their sizes $\left(h_{C}\right)$ in terms of the exchange energies. The remainder of the paper is organized as follows: in Section 2 we describe the basics of our model and method. In Section 3 we present the numerical calculations for magnetization and hysteresis curves and discuss results. Finally, a brief summary is given in Section 4 .

\section{Model and Method}

The magnetic NPs considered have hexagonal lattice structure for any $2 \mathrm{D}$ arrays with radius $R$ related to the numbers of shells and are made of a core surrounded by a surface [30]. A schematic representation of such a structure has been shown in Figure 1 of [31]. We have supposed an Ising spin $\left(S_{i}\right)$ at each lattice site $i$ which takes on the values $\pm 1,0$, as usual $[31,32]$. In order to describe the magnetic properties of the system, we have studied the classical Ising Hamiltonian for a configuration $\left\{S_{i}\right\}$ :

$$
\begin{aligned}
H\left\{S_{i}\right\}= & -J \sum_{\langle i, j\rangle} S_{i} S_{j}-K \sum_{\langle i, j\rangle} S_{i}^{2} S_{j}^{2}-L \sum_{\langle i, j\rangle}\left(S_{i}^{2} S_{j}+S_{i} S_{j}^{2}\right) \\
& -h \sum_{\langle i, j\rangle}\left(S_{i}+S_{j}\right),
\end{aligned}
$$

where $\langle i, j\rangle$ indicates a sum over the nearest neighbors. $J, K$, and $L$ denote, respectively, the dipolar (bilinear), quadrupolar (biquadratic), and odd interactions. In (1), the terms with exchange parameters $J$ and $K$ are even sector contributions while the magnetic field-like perturbations ( $L$ and $h$ ) are odd sector terms [34]. Since the parameter $L$, also called dipolar-quadrupolar interaction, is associated with dipolar and quadrupolar pair interactions, it is combined with $J$ and $K$ via a geometric mean [37] by $L=(J K)^{1 / 2}$. All interaction parameters appearing in (1) are measured in units of $k_{B} T\left(k_{B}\right.$ Boltzmann constant and $T$ temperature) and are kept fixed in computing a phase diagram. In connection with multicritical phenomena the above Hamiltonian has been investigated extensively. In most of the cases considered so far the materials are bulk samples $[35,36,38]$. However, to the best of our knowledge, there are no reports on the magnetic properties of the nanoscale case through the inclusion of dipole-quadrupole interaction in the Hamiltonian which has recently been evidenced in some $C / S$ magnetic NPs using the synthesis techniques [40].

Having defined the relevant spins and Hamiltonian expression that describe the system, we separate Ising spins in three components within the NPs. These are $C, C S$, and $S$ which have the spin numbers denoted by $N_{C}, N_{C S}$, and $N_{S}$, respectively. For the $C / S$-type NPs, we separate (1) into three terms $\left(H_{C}, H_{C S}, H_{S}\right)$ including all interactions between nearest neighboring spins in core $\left(J_{C}, K_{C}, L_{C}\right)$, core-surface $\left(J_{C S}, K_{C S}, L_{C S}\right)$, and surface $\left(J_{S}, K_{S}, L_{S}\right)$ regions. Here $L_{C}=$ $\left(J_{C} K_{C}\right)^{1 / 2}, L_{C S}=\left(J_{C S} K_{C S}\right)^{1 / 2}$, and $L_{S}=\left(J_{S} K_{S}\right)^{1 / 2}$. Using the theoretical formulation based on the PA in Kikuchi version $[41,42]$, the particle's magnetization is easily calculated from the definition:

$$
m=P_{++}+P_{+0}+P_{+-}-\left(P_{-+}+P_{-0}+P_{--}\right),
$$

where the bond variables $\left(P_{i j}, i, j=+, 0,-\right)$ are found from the numerical solutions of the following set of self-consistent equations:

$$
P_{i j}=Z^{-1}\left(p_{i} p_{j}\right)^{(\gamma-1) / \gamma} \exp \left(-\beta \varepsilon_{i j}\right) \equiv Z^{-1} e_{i j} .
$$

Here, $\beta=1 / k_{B} T, p_{i}(i=+, 0,-)$ is the point/state variable, $\gamma$ is the coordination number of a given lattice site inside the $\mathrm{NP}$, and $Z$ is the partition function defined by the relation $Z=\sum_{i, j=+, 0,-} e_{i j}$. For the energy parameters $\varepsilon_{i j}$ in (3) we have recently introduced the definition $[31,32]$

$$
\varepsilon_{i j}=N_{P}^{C} \varepsilon_{i j}^{C}+N_{P}^{C S} \varepsilon_{i j}^{C S}+N_{P}^{S} \varepsilon_{i j}^{S}, \quad(i, j=+, 0,-),
$$

where $N_{P}^{C}=\left(N_{C} \gamma_{C} / 2\right)-N_{C S}, N_{P}^{C S}=2 N_{C S} \gamma_{C S} / 2, N_{P}^{S}=$ $N_{S} \gamma_{S} / 2$ are the numbers of spin pairs; $\gamma_{C}, \gamma_{C S}, \gamma_{S}$ are the coordination numbers; and $\varepsilon_{i j}^{C}, \varepsilon_{i j}^{C S}, \varepsilon_{i j}^{S}$ are called the bond energies of spin pairs $(i, j)$ for $C, C S$, and $S$ ions, respectively. They are computed easily as in Table 1 using (1).

Based on the numerical solutions of (3) using iteration technique, we have firstly obtained the magnetization and hysteresis curves for the smart $C / S$ NPs and investigated their temperature and particle size behaviours in Figures 14, respectively. We have then, in Figure 5, produced and analyzed the calculated data for the odd interaction dependence 
TABLE 1: Bond energies of spin pairs $(i, j)$ for $C, C S$, and $S$ ions.

\begin{tabular}{lccc}
\hline & $C$ & $C S$ & $S$ \\
\hline$\varepsilon_{++}$ & $-J_{C}-K_{C}-2 L_{C}-2 h$ & $-J_{C S}-K_{C S}-2 L_{C S}$ & $-J_{S}-K_{S}-2 L_{S}-2 h$ \\
$\varepsilon_{+0}$ & $-h$ & 0 & $-h$ \\
$\varepsilon_{+-}$ & $J_{C}-K_{C}$ & $J_{C S}-K_{C S}$ & $J_{S}-K_{S}$ \\
$\varepsilon_{0+}$ & $-h$ & 0 & $-h$ \\
$\varepsilon_{00}$ & 0 & 0 & 0 \\
$\varepsilon_{0-}$ & $h$ & 0 & $h$ \\
$\varepsilon_{-+}$ & $J_{C}-K_{C}$ & $J_{S S}-K_{S}$ & $h$ \\
$\varepsilon_{-0}$ & $h$ & 0 & $-J_{S}-K_{S}+2 L_{S}+2 h$ \\
$\varepsilon_{--}$ & $-J_{C}-K_{C}+2 L_{C}+2 h$ & $-J_{C S}-K_{C S}+2 L_{C S}$ &
\end{tabular}

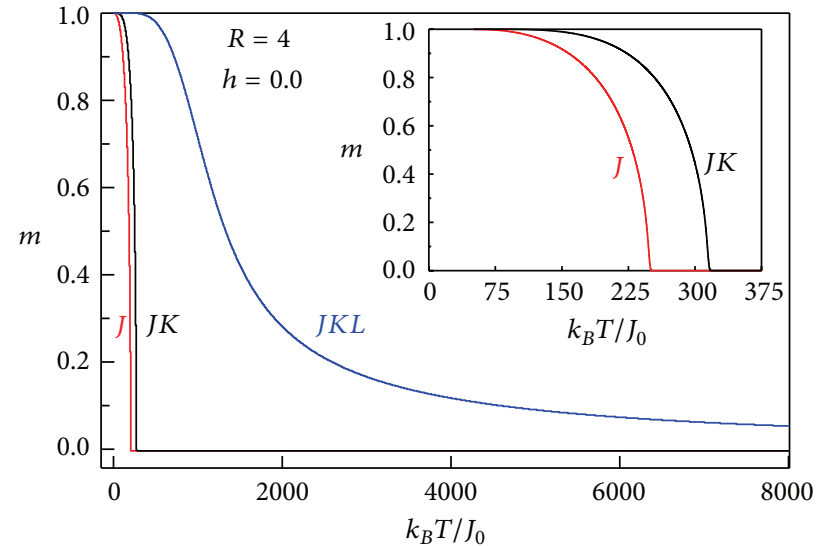

Figure 1: Magnetization $(m)$ versus reduced temperature $\left(k_{B} T / J_{0}\right)$ in the absence $(J$ and $J K)$ and presence $(J K L)$ of odd interaction $(L)$ for $R=4$ and $h=0.0$.

of the hysteresis loops. Finally, effects of the same interaction on the split loops are presented in Figure 6. In all figures, we mainly focus on nonzero odd interactions which enable us to see a number of results numerically resulting in deeper understanding of the behaviour of exchange-biased FM core and FM surface NPs.

\section{Results and Discussion}

We start by discussing the properties of magnetization curves of noninteracting composite NPs, as yielded by the present model Hamiltonian (see (1)). Figure 1 shows the reduced temperature $\left(k_{B} T / J_{0}\right)$ dependence of magnetization $(m)$ for a very small composite nanoparticle with $R=4$ shells under zero magnetic field $(h=0.0)$. In our calculations we have selected, for simplicity, $J_{C}=J_{S}=J_{0}, J_{C S}=-J_{0}, K_{C}=K_{S}=$ $K_{0}, K_{C S}=-K_{0}$, and $L_{C}=L_{C S}=L_{S}=L_{0}$. The curves present three different cases. The red curve, denoted by the letter $J$, corresponds to absence of quadrupolar and odd interactions $\left(J_{0}=1, K_{0}=L_{0}=0\right.$ ), the black curve (or curve $J K$ ) is for the presence of both dipolar and quadrupolar couplings with $J_{0}=K_{0}=1, L_{0}=0$, and the blue one (curve $J K L$ ) is for the presence of all interaction terms, or $J_{0}=K_{0}=L_{0}=1$. When the odd interaction is neglected, the magnetization of the NP decreases continuously from one (1.0) with increasing temperature and converges to zero (0.0). This behaviour is known as the ferromagnetic-paramagnetic (PM) transition in magnetic nanoparticles [43]. The inset of the figure shows that the Curie temperature for the case $J_{0}=1, K_{0}=L_{0}=0$ is less than one for $J_{0}=K_{0}=1, L_{0}=0$. On the other hand, in the presence of $L$ interaction in addition to $J$ and $K$ interactions (or $J_{0}=K_{0}=L_{0}=1$ ), the above transition property has been removed; that is, there is no FM-PM transition and the nanoparticle occupies single magnetic phase $(m \neq 0)$.

Magnetization $(m)$ versus $h$ calculations for a composite nanoparticle with $R=4$ shells are performed at $T=200 J_{0} / k_{B}$ and results are displayed in Figure 2. Three hysteresis curves corresponding to the cases $J, J K$, and $J K L$ in Figure 1 are seen in Figure 2(a). It is pointing out that the hysteresis loop (black curve) is regular and symmetrical around $h=0.0$ when $J_{0}=$ $K_{0}=1, L_{0}=0$. In other words, the values of coercive fields for decreasing and increasing field branches (or the points where the loop intercepts the negative and positive $h$ axis), denoted by $h_{d}$ and $h_{i}$, respectively, are equal with opposite sign $\left(h_{i}=\right.$ $\left.-h_{d}=0.098\right)$. The loop becomes narrower and coercive fields decrease to $h_{i}=-h_{d}=0.032$ under zero biquadratic interaction $\left(K_{0}=0\right)$, plotted as the red curve in the figure. On the other hand, when the odd interaction is present for $J_{0}=K_{0}=L_{0}=1$, one can easily notice that the hysteretic behaviour displays a remarkable property, that is, a large shift $\left(h_{E}\right)$ towards negative magnetic field values with $h_{i}=-0.990$ and $h_{d}=-1.466$, as can be seen from the blue curve in the figure. This property is known as EB effect and determined from the horizontal shift in the midpoint of the loop where it is located at $h=-1.228$. The expression given in the literature $[6,10]$ for the shift $h_{E}$ of any hysteresis loop is $h_{E}=\left|h_{i}+h_{d}\right| / 2$. Based on this expression, the exchange field reads $h_{E}=1.228$. We also observe an increase in the coercive field $\left(\Delta h_{C}=\right.$ $0.140)$ with $h_{C}=0.238$ according to the definition $h_{C}=$ $\left|h_{i}-h_{d}\right| / 2[6,10]$. Such a shift accompanied by a coercivity enhancement $\left(\Delta h_{C}\right)$ has been generally observed in metallic magnetic NPs [6], nanogranular materials [7], and some nanocomposite systems [14] characterized by the coexistence of different exchange-coupled magnetic phases. Another important property which originates from nonzero $L_{0}$ values is the asymmetry of the loop. It is an intrinsic effect which develops with increasing values of interface coupling for the EB magnetic structures [44-46]. 


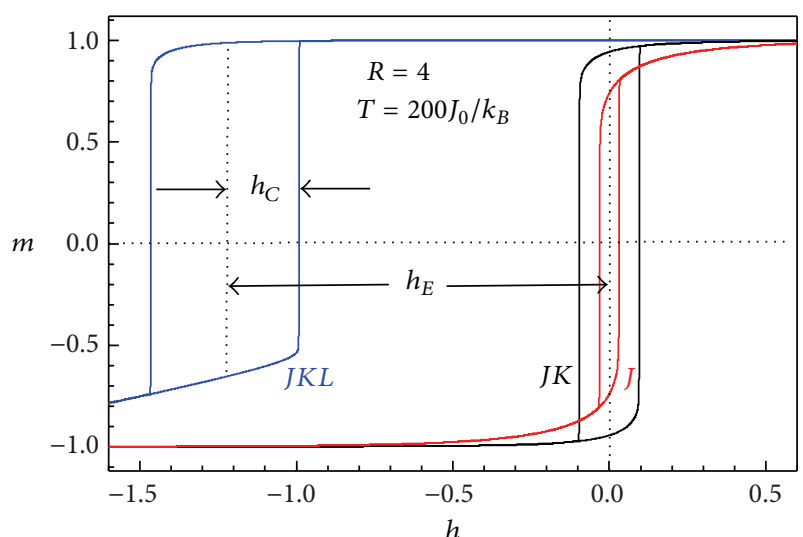

(a)

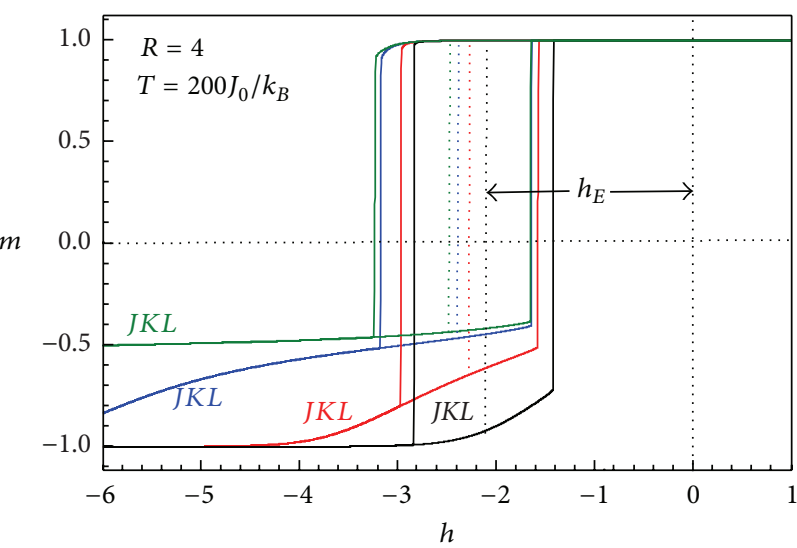

(b)

Figure 2: (a) $m$ - $h$ hysteresis loops for a composite NP in the absence ( $J$ and $J K$ ) and presence $(J K L)$ of odd interaction $(L)$. (b) Hysteresis loops $(J K L)$ as in (a), but using different exchange parameters; $R=4 ; T=200 J_{0} / k_{B}$.

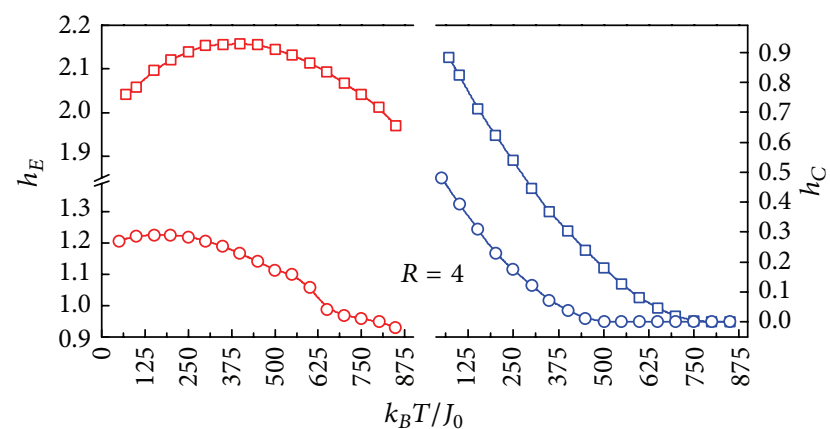

(a)

(b)

FIGURE 3: Reduced temperature $\left(k_{B} T / J_{0}\right)$ dependence of (a) exchange-bias field $\left(h_{E}\right)$ and (b) coercive field $\left(h_{C}\right)$. Empty circles and squares correspond to the cases $J_{0}=K_{0}=L_{0}=1, J_{0}=K_{0}=$ $L_{0}=2$, respectively; $R=4$.

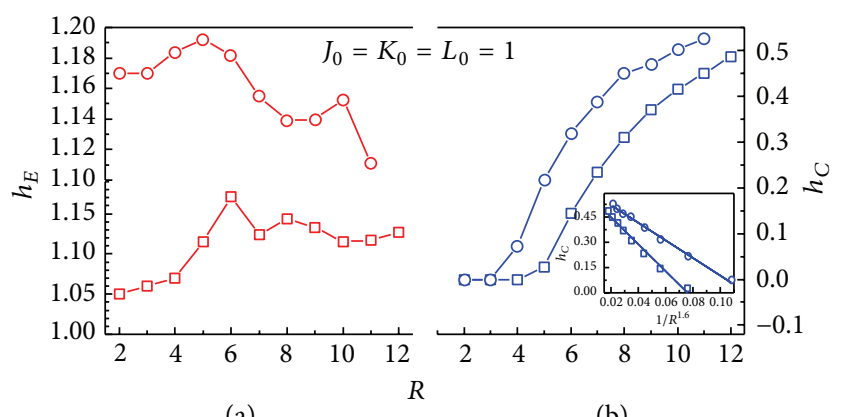

(a)

(b)

FIGURE 4: Variation of (a) exchange-bias field $\left(h_{E}\right)$ and (b) coercive field $\left(h_{C}\right)$ with particle size $(R)$. Empty circles and squares correspond to the cases $T=350 J_{0} / k_{B}, T=700 J_{0} / k_{B}$, respectively; $J_{0}=K_{0}=L_{0}=1$.

For the sake of comparison, we have also calculated the hysteresis for the same system, but using different interface exchange parameters, whose results are drawn in Figure 2(b). Again we have considered four-shell NPs but with the following characteristics: the exchange interactions in the

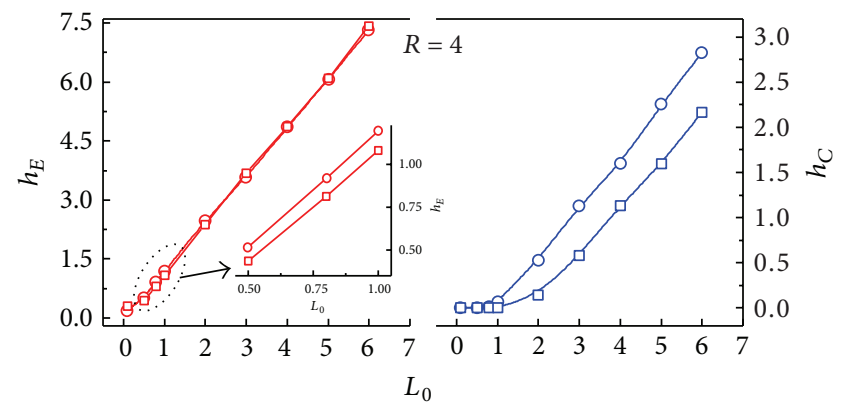

(a)

(b)

FIgURE 5: Odd interaction $\left(L_{0}\right)$ variations of (a) $h_{E}$ and (b) $h_{C}$. Empty circles and squares correspond to the cases $T=350 J_{0} / k_{B}$, $T=700 J_{0} / k_{B}$, respectively; $R=4$.

core region $(R=0-3)$ are given by simple FM coupling values $J_{C}=K_{C}=L_{C}=1$, the outermost shell (or surface) $(R=4)$ is also FM with $J_{S}=K_{S}=L_{S}=5$, and the couplings between $R=3$ and $R=4$ shells are due to AFM dipolar and quadrupolar couplings with $J_{C S}=K_{C S}=-1,-2,-4,-8$ and FM odd interactions with $L_{C S}=1,2,4,8$ as shown by the black, red, blue, and green curves, respectively. As evidenced by all colored curves in this graph, the shifted loops due to nonzero odd interaction $\left(L_{C S}\right)$ present another clear exchange shift related to the increase of interfacial AFM dipolar and quadrupolar coupling values $\left(J_{C S}, K_{C S}\right)$ from -1 to -8 . Also, on increasing these couplings, the loops become more asymmetric in shape than one with smaller couplings. In other words, we observe a large curvature at the bottom of the black $m$ - $h$ loop along the magnetic field axis in the negative direction while it changes into crookedness with increasing $L_{C S}$ values (Figure 2(b)). In upward curvature/crookedness case, the magnitude in the negative magnetization $(-m)$ direction is smaller than one when it is in the opposite $(+m)$ direction. Origin of abovementioned asymmetric properties is related to the upward vertical shear of $m$ - $h$ loops by Ceylan et al. [47, 48]. In [47], 


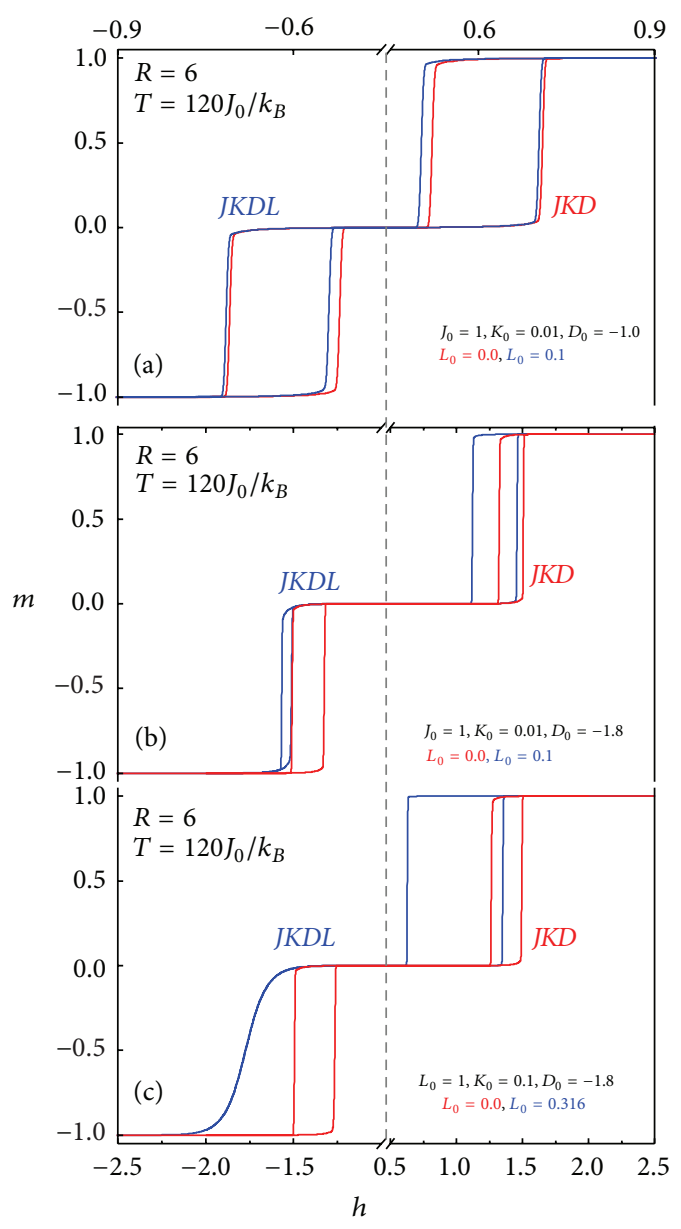

FIGURE 6: (a) Split $m$ - $h$ hysteresis loops for a composite NP in the absence $(J K D)$ and presence $(J K D L)$ of odd interaction $\left(L_{0}\right)$. (b) and (c) Hysteresis loops $(J K D L)$ as in (a), but using different exchange parameters; $R=6, T=120 J_{0} / k_{B}$.

the crookedness was explained as originating in the pinned spins at the interface between the core and shell regimes of the NPs. Some parts of the interface spins between the core and surface cannot rotate with the field due to the pinned spins in the negative magnetization direction. The number of pinned spins is a measure of the curvature/crookedness at the bottom of the $m$ - $h$ loops with EB effects. Furthermore, the EB effects are correctly related to the decreasing of the number of the pinned spins at the interface. To understand the behaviour in the negative magnetization direction we need to investigate separately positive and negative magnetization characteristics. This can be achieved through use of the $m-h$ loops split according to the magnetization direction. The split $m$ - $h$ loops were recently investigated in relation to the single-ion anisotropy parameter $(D)$ based on a similar model calculation with $D_{C}=D_{S}=D_{0}[31,32]$. Effects of the odd interaction on the split loops will be shown in the last figure and discussed in the last paragraph of this section.

The reduced temperature dependencies of $h_{E}$ and $h_{C}$ obtained from magnetic hysteresis loops ( $m$ versus $h$ ) in the temperature range from 50 to 850 for the NP with size $R=4$ were studied using the interactions $J_{0}=K_{0}=L_{0}=1$ (empty circles with red and blue colors) and $J_{0}=K_{0}=L_{0}=2$ (empty squares with red and blue colors), as shown in Figure 3. One can see from the figure that the amount of hysteretic shift for the case $J_{0}=K_{0}=L_{0}=1$ increases gradually with increasing temperature and remains constant until the temperature approaches $T \approx 266 J_{0} / k_{B}$, where $h_{E}$ starts to decrease (Figure 3(a)), whereas the width of the hysteresis decays exponentially and the loop becomes narrower until it vanishes when the temperature rises to $T \approx 500 J_{0} / k_{B}$ (Figure 3(b)). Another exchange field value calculated for $J_{0}=K_{0}=L_{0}=2$ displays a similar behaviour for the temperature dependence with plateau between temperatures 250 and 375 , but it jumps to larger values at each temperature, as compared to one for the case $J_{0}=K_{0}=L_{0}=1$. Again we observed exponential decay in the coercivity with increasing temperature below $T \approx 750 J_{0} / k_{B}$, where it goes to zero, also seen in Figure 3(b). But the decay is much slower than the previous case. The exponential decrease of $h_{C}$ with temperature for a fixed particle size agrees with the same behaviour that was observed experimentally for the monodisperse NPs in $[15,49]$ as well as the simulated composite NPs [20]. Remarkably, the observed temperature dependence of $h_{E}$ resembles more those reported in previous experimental investigations on nanometric iron particles embedded in an AFM matrix [5] and on heavily oxidized core-shell NPs [9] rather than the ones in monodisperse systems. As can be seen in Figure 3(b), the system also exhibits a coercivity enhancement, $\Delta h_{C}$, from $h_{C}$ at $J_{0}=K_{0}=L_{0}=1$ to $h_{C}$ at $J_{0}=$ $K_{0}=L_{0}=2$.

To demonstrate the role of particle size, we keep the temperature constant and we change the radius of the NP described in Figures 1 and 2(a). Our results for $T=350 J_{0} / k_{B}$ (empty circles with red and blue colors) and $T=700 J_{0} / k_{B}$ (empty squares with red and blue colors) are presented in Figure 4 where we have plotted $h_{E}$ and $h_{C}$ as a function of $R$. In this case we have started with $J_{0}=K_{0}=L_{0}=1$. It can be seen, from Figure $4(\mathrm{a})$, that $h_{E}$ at $T=350 J_{0} / k_{B}$ increases first, reaches its maximum around $R=5$, and then decreases with increasing particle size. In addition, another peak with a lower height appears at $R=10$. With increasing temperature $\left(T=700 J_{0} / k_{B}\right), h_{E}$ decreases and the peak positions change to $R=6$ and 8 . Although the reduction of the loop shift with $R$ confirms similar trends observed experimentally [10], a closer inspection of our results reveals the notable oscillations [11] of $h_{E}$ as function of $R$. However, it is clear that with increasing radius $h_{C}$ at $T=350 J_{0} / k_{B}$ also grows but it reduces when the temperature is raised to $T=700 J_{0} / k_{B}$ in Figure $4(\mathrm{~b})$. For both temperatures, the functional relation $h_{C} \approx R^{-1.6}$ fits very well the results of our coercivity calculations for the NPs of sizes $2 \leq R \leq 12$ as shown in the inset of Figure 4(b). The linear relation described above was also obtained by Rego and Figueiredo [30]. Our observed size dependence of the EB and coercivity can be explained on the basis of the models offered in [50]. In general, the decrease of the EB is due to the smallness of the volume-dependent Zeeman energy and the decrease of the coercivity is related to the surface anisotropy. For the nanosized regime (say $<\sim 10 \mathrm{~nm}$, superparamagnetic limit), Zeeman energy may be less than the anisotropy energy 
and both effects may contribute to the slimming of the EB and coercivity for very small particles [51]. But Zeeman energy and anisotropy energy are both normally proportional to the volume when the size is greater than $\sim 10 \mathrm{~nm}$. We indicate from these observations that $h_{E}$ and $h_{C}$ are strongly related to the particle size, which was previously reported in experiments for the polycrystalline NPs [10].

In Figure 5, we have investigated the role played by the odd interaction strength $\left(L_{0}\right)$ on the observations of $h_{E}$ and $h_{C}$ by comparing the calculations for two different temperatures with the same particle radius $R=4$. In the figure, empty circles correspond to the temperature $T=$ $350 J_{0} / k_{B}$ while empty squares are for another temperature, that is, $T=700 J_{0} / k_{B} . h_{E}$ shows a linear change with $L_{0}$ values when $L_{0}>1$ (Figure 5(a)). But there is no clear difference between two calculated pieces of data for two temperatures. According to the behaviour demonstrated in the inset of Figure 5(a), a slight change for 350 and $700 J_{0} / k_{B}$ of temperatures occurs at very small $L_{0}$ values with $L_{0} \leq 1$. This small difference for $h_{E}$ is attributed to the decreasing of the interactions between pinned spins at the $C / S$ interface. On the other hand, the field $h_{C}$ shows nearly the same linear behaviour as $h_{E}$ for $L_{0}>2$. Two coercive fields calculated using both temperatures $\left(T=350 J_{0} / k_{B}, T=700 J_{0} / k_{B}\right)$ at the same $L_{0}$ values are different from each other while especially $h_{C}$ exhibits small slope for $L_{0}<2$. Furthermore, differences of $h_{C}$ for 350 and $700 J_{0} / k_{B}$ of temperatures disappear at each $L_{0}$ when $L_{0}<1$. In this case, the EB gets destabilized in the smaller $L_{0}$ due to the thermal fluctuations of the $C$ and $S$ spins.

Finally, we have shown in Figure 6 further calculations performed at a different temperature $\left(T=120 J_{0} / k_{B}\right)$ for a bigger composite nanoparticle with $R=6$ shells, which obviously indicates the effects of the interaction $L_{0}$ on the split hysteresis curves or double FM loops. Detailed discussion of these loops was given in [31, 32]. A split $m-h$ hysteresis loop for a nanoparticle is observed in the presence of single-ion anisotropy parameter $(D)$. A similar curve is reproduced here for comparison using $J_{0}=1, K_{0}=0.01$, $D_{0}=-1.0, L_{0}=0.0$, illustrated as the red curve (or curve $J K D$ ) in Figure 6(a). When the odd interaction is present in addition to $D$ with $J_{0}=1, K_{0}=0.01, D_{0}=-1.0, L_{0}=0.1$, the hysteresis loop (curve $J K D L$ or blue curve) is also splitting in double FM loops but with a small loop shift towards negative magnetic field direction $(-h)$, also indicated in Figure 6(a). Figure 6(b) shows another double FM loop structure at $J_{0}=$ $1, K_{0}=0.01, D_{0}=-1.8, L_{0}=0.1$ (blue curve). On increasing $L_{0}$ from 0.0 to 0.1 , one of the split loops becomes narrower while the other is wider than the red one for $L_{0}=0.0$. On the other hand, when increasing both $K_{0}$ and $L_{0}$ values, that is, $J_{0}=1, K_{0}=0.1, D_{0}=-1.8, L_{0}=0.316$, the FM loop observed at negative magnetization side disappears and the particle shows a single line hysteresis loop at this side but we can still see only one FM loop at positive magnetization side (see also the blue curve in Figure 6(c)). This single line hysteresis loop may originate from the same direction pinned spins at $C / S$ interface.

\section{Summary and Conclusion}

In summary, we have presented a spin-1 Ising model for the composite $C / S$ NPs that have revealed a shift (also known as the EB effect) in the hysteresis loops. The effect was found to increase with dipolar-quadrupolar interaction strength. The coercivity is also sensitive to this interaction parameter. In contrast to FM/AFM multilayers [27], we have concluded that the behaviours of the fields $h_{E}$ and $h_{C}$ are of the same origin; both are present and they are strongly correlated. We have also found usual temperature and size dependencies of EB and coercivity values. However, when the odd interaction is absent, the hysteresis loops of $C / S \mathrm{NPs}$ are always symmetric, which is independent of temperature below the critical temperature. The occurrence of asymmetry in the loops is also linked with the appearance of the maximum of EB, increasing for larger anisotropy ratios, as has been found in other EB systems for smaller particles [44, 45]. In other words, by a unidirectional shift of the $m-h$ curves and by increasing coercivity with decreasing temperatures, one finds good conditions to observe such an asymmetry in the loops correlated with nonzero odd interaction strength. While no vertical shift in the hysteresis is observed, all asymmetric loops obtained in this study exhibit an upward curvature/crookedness shape as well as a horizontal shift. As follows from the experiments on core/shell structured NPs $[47,48]$ the presence of this type of asymmetry accompanying the EB has been associated with the presence of pinned spins located at the $C / S$ interface within the NPs. In our NPs, we have also observed asymmetry effects on the split hysteresis loops which suggest that there is a variation of pinning strength amongst the interface spins depending on the particle size and temperature [50].

In conclusion, the inclusion of odd sector contributions in the Ising Hamiltonian for a single composite nanoparticle induces above significant hysteresis properties. Our investigations show also that in general one has to deal with different interaction strengths for the hysteretic behaviours of various nanostructured materials, resulting in interesting and even surprising new effects. Hence, it is possible to control the coercivity and the EB as well as the asymmetry during magnetization reversal processes along the hysteresis loops of any given nanosystem. For example, the present theoretical approach provides a model system for studying magnetic properties within the periodic arrays of NPs with interparticle dipole-quadrupole interactions [52]. In future, we expect a deeper insight into the physics of EB from a comparison of the ideas from [52] and our calculations.

\section{Competing Interests}

The authors declare that they have no competing interests.

\section{Acknowledgments}

This work was supported by the Scientific Research Projects Coordination Unit of Akdeniz University. 


\section{References}

[1] W. H. Meiklejohn and C. P. Bean, "New magnetic anisotropy," Physical Review, vol. 102, no. 5, pp. 1413-1414, 1956.

[2] M. Takahashi, A. Yanai, S. Taguchi, and T. Suzuki, "Study of exchange anisotropy in co-coo evaporated thin films," Japanese Journal of Applied Physics, vol. 19, no. 6, pp. 1093-1106, 1980.

[3] H. Morita, H. Hiroyoshi, and K. Fukamichi, "Field cooling effect on magnetic anisotropy of amorphous $\mathrm{Fe}_{91.4} \mathrm{Zr}_{86}$ alloy," Journal of Physics F: Metal Physics, vol. 16, no. 4, pp. 507-513, 1986.

[4] A. Petrov, I. Kudrenitskis, S. Belogurov, and S. Nepijko, "Exchange anisotropy of cobalt fine particles," Annalen der Physik, vol. 5, no. 8, pp. 627-632, 1996.

[5] J. Sort, V. Langlais, S. Doppiu et al., "Exchange bias effects in Fe nanoparticles embedded in an antiferromagnetic $\mathrm{Cr}_{2} \mathrm{O}_{3}$ matrix," Nanotechnology, vol. 15, no. 4, pp. S211-S214, 2004.

[6] U. Wiedwald, J. Lindner, M. Spasova, Z. Frait, and M. Farle, "Effect of an oxidic overlayer on the magnetism of Co nanoparticles," Phase Transitions, vol. 78, no. 1-3, pp. 85-104, 2005.

[7] Ò. Iglesias, A. Labarta, and X. Batlle, "Exchange bias phenomenology and models of core/shell nanoparticles," Journal of Nanoscience and Nanotechnology, vol. 8, no. 6, pp. 2761-2780, 2008.

[8] B. Kalska, P. Fumagalli, M. Hilgendorff, and M. Giersig, "Co/ CoO core-shell nanoparticles-temperature-dependent magneto-optic studies," Materials Chemistry and Physics, vol. 112, no. 3, pp. 1129-1132, 2008.

[9] S. E. Inderhees, J. A. Borchers, K. S. Green et al., "Manipulating the magnetic structure of $\mathrm{Co}$ core/CoO shell nanoparticles: implications for controlling the exchange bias," Physical Review Letters, vol. 101, no. 11, Article ID 117202, 2008.

[10] X. H. Huang, J. F. Ding, G. Q. Zhang, Y. Hou, Y. P. Yao, and X. G. Li, "Size-dependent exchange bias in $\mathrm{La}_{0.25} \mathrm{Ca}_{0.75}$ $\mathrm{MnO}_{3}$ nanoparticles," Physical Review B-Condensed Matter and Materials Physics, vol. 78, no. 22, Article ID 224408, 2008.

[11] Ò. Iglesias, X. Batlle, and A. Labarta, "Particle size and cooling field dependence of exchange bias in core/shell magnetic nanoparticles," Journal of Physics D: Applied Physics, vol. 41, no. 13, Article ID 134010, 2008.

[12] Q. K. Ong, A. Wei, and X.-M. Lin, "Exchange bias in $\mathrm{Fe} / \mathrm{Fe}_{3} \mathrm{O}_{4}$ core-shell magnetic nanoparticles mediated by frozen interfacial spins," Physical Review B, vol. 80, no. 13, Article ID 134418, pp. 1-6, 2009.

[13] S. Guo, W. Liu, H. Meng et al., "Exchange bias and its training effect in $\mathrm{Ni} / \mathrm{NiO}$ nanocomposites," Journal of Alloys and Compounds, vol. 497, no. 1-2, pp. 10-13, 2010.

[14] S. Laureti, D. Peddis, L. Del Bianco et al., "Exchange bias and magnetothermal properties in Fe@Mn nanocomposites," Journal of Magnetism and Magnetic Materials, vol. 324, no. 21, pp. 3503-3507, 2012.

[15] Y. Hwang, S. Angappane, J. Park, K. An, T. Hyeon, and J.-G. Park, "Exchange bias behavior of monodisperse $\mathrm{Fe}_{3} \mathrm{O}_{4} / \gamma-\mathrm{Fe}_{2} \mathrm{O}_{3}$ core/shell nanoparticles," Current Applied Physics, vol. 12, no. 3 , pp. 808-811, 2012.

[16] T. Maity, S. Goswami, D. Bhattacharya, and S. Roy, "Origin of the asymmetric exchange bias in $\mathrm{BiFeO}_{3} / \mathrm{Bi}_{2} \mathrm{Fe}_{4} \mathrm{O}_{9}$ nanocomposite," Physical Review B-Condensed Matter and Materials Physics, vol. 89, no. 14, Article ID 140411, 2014.

[17] D. R. Saha, A. K. Nandi, and D. Chakravorty, "Exchange bias effect in composites of cuo nanoparticles and nanosilica glass," Journal of Magnetism and Magnetic Materials, vol. 355, pp. 184187, 2014.
[18] Ò. Iglesias, X. Batlle, and A. Labarta, "Microscopic origin of exchange bias in core/shell nanoparticles," Physical Review B, vol. 72, no. 21, Article ID 212401, pp. 1-4, 2005.

[19] Ò. Iglesias and A. Labarta, "Monte Carlo simulation study of exchange biased hysteresis loops in nanoparticles," Physica B: Condensed Matter, vol. 372, no. 1-2, pp. 247-250, 2006.

[20] M. Vasilakaki and K. N. Trohidou, "Numerical study of the exchange-bias effect in nanoparticles with ferromagnetic core/ferrimagnetic disordered shell morphology," Physical Review B-Condensed Matter and Materials Physics, vol. 79, no. 14, Article ID 144402, 2009.

[21] Y. Hu, Y. Liu, and A. Du, "Effect of cooling field strength and ferromagnetic shell shape on exchange bias in nanoparticles with inverted ferromagnetic-antiferromagnetic core-shell morphology," Physica Status Solidi B, vol. 247, no. 4, pp. 972-978, 2010.

[22] R. F. L. Evans, D. Bate, R. W. Chantrell, R. Yanes, and O. Chubykalo-Fesenko, "Influence of interfacial roughness on exchange bias in core-shell nanoparticles," Physical Review B, vol. 84, no. 9, Article ID 092404, pp. 1-4, 2011.

[23] Y. Hu and A. Du, "Surface-anisotropy and training effects of exchange bias in nanoparticles with inverted ferromagneticantiferromagnetic core-shell morphology," Journal of Applied Physics, vol. 110, no. 3, Article ID 033908, 2011.

[24] Y. Hu, Y. Liu, A. Du, and F. Shi, "Dependence of exchange bias on core/shell relative dimension in ferromagnetic/ antiferromagnetic nanoparticles," Physics Letters, Section A: General, Atomic and Solid State Physics, vol. 378, no. 22-23, pp. 1667-1674, 2014.

[25] C. Binek, B. Kagerer, S. Kainz, and W. Kleemann, "Exchange bias in $\mathrm{FeF}_{2}-\mathrm{Co} / \mathrm{Pt}$ heterosystems with perpendicular anisotropy," Journal of Magnetism and Magnetic Materials, vol. 226-230, pp. 1814-1816, 2001.

[26] B. Negulescu, R. Tanasa, and A. Stancu, "Ising model for exchange bias in ferromagnetic/antiferromagnetic bilayers," Journal of Optoelectronics and Advanced Materials, vol. 6, no. 3, pp. 991-994, 2004.

[27] G. Scholten, K. D. Usadel, and U. Nowak, "Coercivity and exchange bias of ferromagnetic/antiferromagnetic multilayers," Physical Review B, vol. 71, no. 6, Article ID 064413, pp. 1-7, 2005.

[28] T. Błachowicz, "Simulating a perpendicular exchange-biased structure with a partially covering nonmagnetic insertion at the FM/AFM interface," Central European Journal of Physics, vol. 4, no. 3, pp. 331-340, 2006.

[29] J. Nogués and I. K. Schuller, "Exchange bias," Journal of Magnetism and Magnetic Materials, vol. 192, no. 2, pp. 203-232, 1999.

[30] L. G. C. Rego and W. Figueiredo, "Magnetic properties of nanoparticles in the Bethe-Peierls approximation," Physical Review B-Condensed Matter and Materials Physics, vol. 64, no. 14, Article ID 144424, 2001.

[31] O. Yalçın, R. Erdem, and S. Özüm, "Origin of the martensitic and austenitic phase transition in core-surface smart nanoparticles with size effects and hysteretic splitting," Journal of Applied Physics, vol. 115, no. 5, Article ID 054316, pp. 1-7, 2014.

[32] S. Özüm, O. Yalçın, R. Erdem, H. Bayrakdar, and H. N. Eker, "Martensitic and austenitic transformations in core-surface cubic nanoparticles," Journal of Magnetism and Magnetic Materials, vol. 373, pp. 217-221, 2015.

[33] M. Blume, V. J. Emery, and R. B. Griffiths, "Ising model for the $\lambda$ transition and phase separation in $\mathrm{He}^{3}-\mathrm{He}^{4}$ mixtures," Physical Review A, vol. 4, no. 3, pp. 1071-1077, 1971. 
[34] A. N. Berker and M. Wortis, "Blume-Emery-Griffiths-Potts model in two dimensions: phase diagram and critical properties from a position-space renormalization group," Physical Review $B$, vol. 14, no. 11, pp. 4946-4963, 1976.

[35] Y. Saito, "Spin-1 antiferromagnetic Ising model. I. Bulk phase diagram for a binary alloy," The Journal of Chemical Physics, vol. 74, no. 1, pp. 713-720, 1980.

[36] B.-L. Gu, J. Ni, and J.-L. Zhu, "Structure of the alloy $(\mathrm{GaAs})_{1-\mathrm{x}} \mathrm{Ge}_{2 \mathrm{x}}$ and its electronic properties," Physical Review $B$, vol. 45, no. 8, pp. 4071-4076, 1992.

[37] C. Temirci, A. Kökçe, and M. Keskin, "Equilibrium properties of a spin-1 Ising system with bilinear, biquadratic and odd interactions," Physica A: Statistical Mechanics and Its Applications, vol. 231, no. 4, pp. 673-686, 1996.

[38] A. Lopatnikova and A. N. Berker, "Renormalization-group study of superfluidity and phase separation of helium mixtures immersed in a nonrandom aerogel," Physical Review B, vol. 55, no. 6, pp. 3798-3802, 1997.

[39] D. P. Snowman, "A renormalization-group analysis of a spin1 Ising ferromagnet with competing crystal-field and repulsive biquadratic interactions," Journal of Magnetism and Magnetic Materials, vol. 321, no. 19, pp. 3007-3013, 2009.

[40] D. Yang, G. Li, X. Kang et al., "Room temperature synthesis of hydrophilic $\mathrm{Ln}^{3+}$-doped $\mathrm{KGdF}_{4}(\mathrm{Ln}=\mathrm{Ce}, \mathrm{Eu}, \mathrm{Tb}, \mathrm{Dy})$ nanoparticles with controllable size: energy transfer, size-dependent and color-tunable luminescence properties," Nanoscale, vol. 4, no. 11, pp. 3450-3459, 2012.

[41] R. Kikuchi, "Superposition approximation and natural iteration calculation in cluster-variation method," The Journal of Chemical Physics, vol. 60, no. 3, pp. 1071-1080, 1974.

[42] A. Erdinç and M. Keskin, "Equilibrium and nonequilibrium behavior of the spin-1 Ising model in the quadrupolar phase," Physica A: Statistical Mechanics and Its Applications, vol. 307, no. 3-4, pp. 453-468, 2002.

[43] H. Amekura, Y. Fudamoto, Y. Takeda, and N. Kishimoto, "Curie transition of superparamagnetic nickel nanoparticles in silica glass: a phase transition in a finite size system," Physical Review $B$, vol. 71, no. 17, Article ID 172404, 4 pages, 2005.

[44] B. Beckmann, U. Nowak, and K. D. Usadel, "Asymmetric reversal modes in ferromagnetic/antiferromagnetic multilayers," Physical Review Letters, vol. 91, Article ID 187201, 2003.

[45] J. Camarero, J. Sort, A. Hoffmann et al., "Origin of the asymmetric magnetization reversal behavior in exchange-biased systems: competing anisotropies," Physical Review Letters, vol. 95, no. 5, Article ID 057204, pp. 1-4, 2005.

[46] Ò. Iglesias, X. Batlle, and A. Labarta, "Exchange bias and asymmetric hysteresis loops from a microscopic model of core/shell nanoparticles," Journal of Magnetism and Magnetic Materials, vol. 316, no. 2, pp. 140-142, 2007.

[47] A. Ceylan, C. C. Baker, S. K. Hasanain, and S. I. Shah, "Effect of particle size on the magnetic properties of core-shell structured nanoparticles," Journal of Applied Physics, vol. 100, no. 3, Article ID 034301, 5 pages, 2006.

[48] L. C. Barnsley, Exchange bias in manganese alloys with mixed magnetic behaviour [Ph.D. thesis], Griffith University, Queensland, Australia, 2012.

[49] E. Tronc, M. Noguès, C. Chanéac et al., "Magnetic properties of $\gamma-\mathrm{Fe}_{2} \mathrm{O}_{3}$ dispersed particles: size and matrix effects," Journal of Magnetism and Magnetic Materials, vol. 272-276, pp. 1474-1475, 2004.
[50] A. Mumtaz, K. Maaz, B. Janjua, S. K. Hasanain, and M. F. Bertino, "Exchange bias and vertical shift in $\mathrm{CoFe}_{2} \mathrm{O}_{4}$ nanoparticles," Journal of Magnetism and Magnetic Materials, vol. 313, no. 2, pp. 266-272, 2007.

[51] A. N. Dobrynin, D. N. Ievlev, K. Temst et al., "Critical size for exchange bias in ferromagnetic-antiferromagnetic particles," Applied Physics Letters, vol. 87, no. 1, Article ID 012501, 3 pages, 2005.

[52] A. B. Evyyukhin, C. Reinhardt, U. Zyweietz, and B. N. Chichkov, "Collective resonances in metal nanoparticle arrays with dipole-quadrupole interactions," Physical Review B, vol. 85, no. 24, Article ID 245411, 2012. 

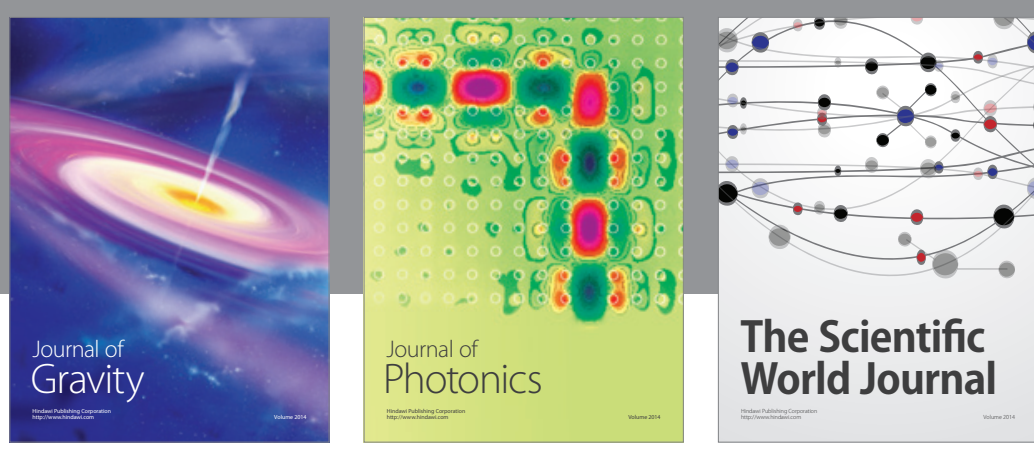

The Scientific World Journal
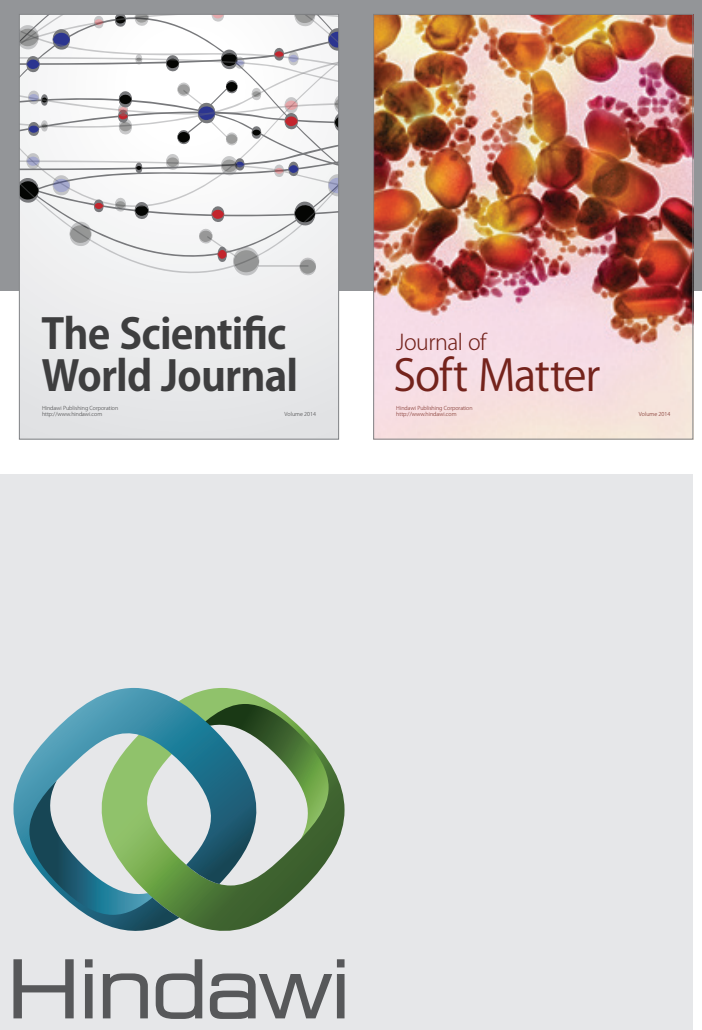

Submit your manuscripts at

http://www.hindawi.com

nternational Journal of

Statistical Mechanics
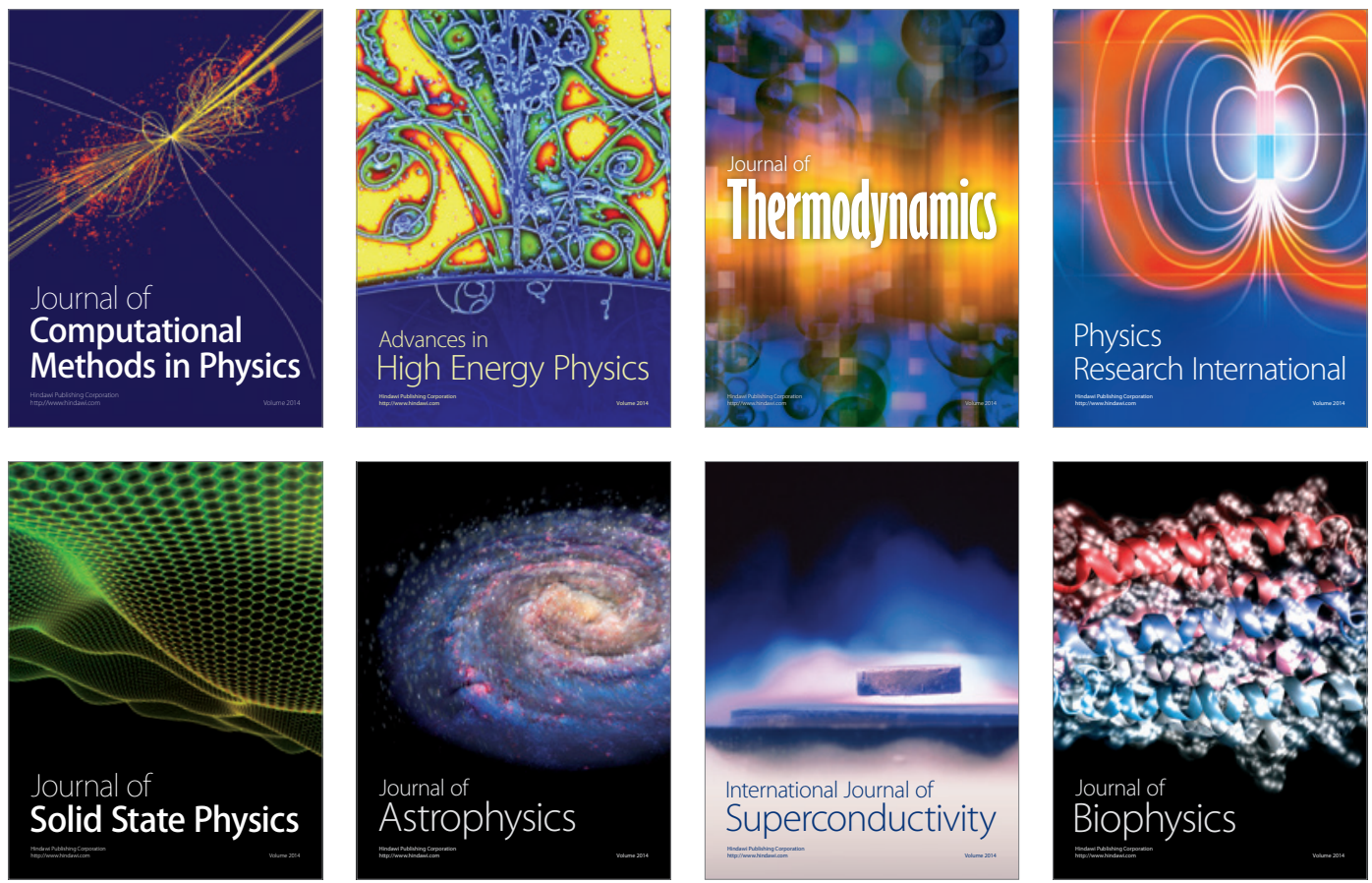
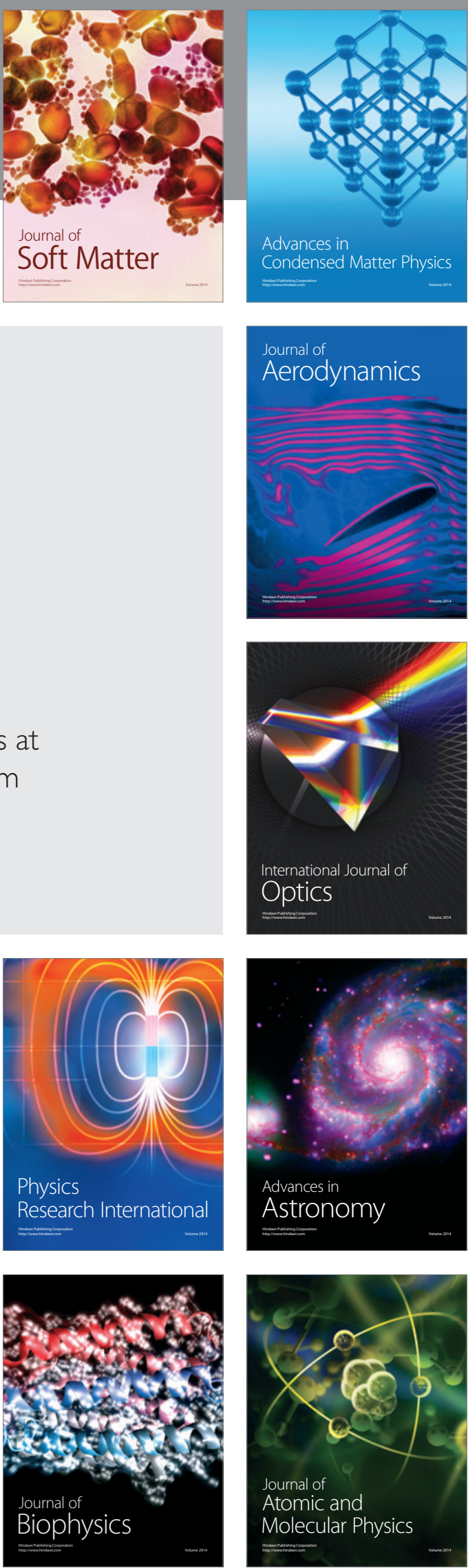